\section{Meio urbano e doenças cardiovasculares}

Trabalho apresentado no Ciclo de Debates sobre Meio-Ambiente, promovido pela FEEMA /SBPC, Rio, 6/82.

\section{Eduardo de Azeredo Costa* Carlos Henrique Klein **}

São discutidas quatro crenças muito comuns em relação às doenças cardiovasculares, especialmente as de que são danos resultantes da urbanização e que são próprias das pessoas ricas. Porém, baseados na análise dos resultados obtidos pelo grupo de estudos dos autores em pesquisas sobre hipertensão arterial, feitas no Rio Grande do Sul e em Volta Redonda, conclui-se que é a maior ou menor proporção de pessoas assalariadas nos setores secundário e terciário que determina o nivel de hipertensão em uma população. E que é a concentração de riquezas, a facilidade de acesso aos bens materiais e/ou a submissão e a repressão na vida social dos individuos que influenciam decisivamente a pressão arterial de cada um.

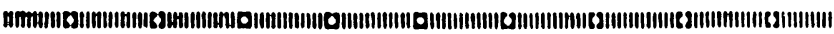

Existem quatro crenças altamente difundidas em relação às doenças cardiovasculares:

1 - que são doenças próprias das pessoas mais ricas;

2 - que são doenças de países desenvolvidos;

3 - que são doenças não-redutíveis, isto é, são uma fatalidade ligada ao envelhecimento das pessoas;

4 - que são doenças da urbanização.

Esse conjunto de crenças leva não só a um imobilismo sanitário, como também tem levado a um outro conjunto de crenças causais, que vão desde o excesso de preocupação pelas responsabilidades dos ricos até o estresse do cotidiano urbano, passando pelo medo e tensão frente ao novo, ao desconhecido.

Tal conjunto de crenças poderia ser facilmente testado, se as estatísticas dos países subdesenvolvidos não fossem tão falhas e se, nas áreas rurais e entre os grupos menos privilegiados das populações, não fossem os recursos médicos mais escassos. Aliás, esses são motivos que podem determinar os achados que parecem confirmar aquelas crenças. Por isso, esses dados devem ser submetidos a severas críticas.
* Secretaria de Estado de Saúde e Higiene do Rio de Janeiro e Escola Nacional de Saúde Pública - FIOCRUZ.RJ.

**Escola Nacional de Saúde Pública - FIOCRUZ-RJ.
Recebido para publicação em $10 / 06 / 85$. 
A Grã-Bretanha é um dos poucos países do mundo capitalista em que o acesso aos serviços de saúde é não apenas universal, mas também de qualidade bastante homogênea, tanto em relação à dicotomia urbano-rural, como entre os grupos sociais. Uma recente e cuidadosa revisão da mortalidade por classe social feita por Rose e Marmot ${ }^{10}$, na Inglaterra e Gales, mostra que as doenças cardiovasculares são, de fato, mais freqüentes nas classes trabalhadoras. Um outro estudo dos mesmos autores ${ }^{8}$, agora em funcionários públicos, mostra que a morbidade por doença coronariana e hipertensão é menor nos administradores e profissionais de nível superior do que nos funcionários não-especializados e menos graduados.

Por outro lado, um estudo especial, patrocinado pela Organização Pan-Americana da Saúde, sobre as características da mortalidade urbana em cidades selecionadas das Américas ${ }^{9}$ mostra que São Paulo, Ribeirão Preto (as duas do Brasil incluídas no estudo) e La Plata (Argentina) apresentavam mortalidade por doenças cardiovasculares bastante próximas das de San Francisco (EUA) e Bristol (Inglaterra).

As duas causas mais importantes de morte dentro do grupo das cardiovasculares são as doenças coronarianas e as cérebro-vasculares. Repare-se também que, no estudo da OPAS aqui referido, ocorre uma inversão de ordem na importância desses grupos de causa: em nosso meio, as cérebro-vasculares são mais freqüentes, enquanto que as coronarianas o são em São Francisco e Bristol.

No entanto, é preciso lembrar que as tendências de mortalidade nesse século, nos países desenvolvidos, podem não apenas explicar esse achado, como também derrubar outra crença que mencionamos no início, qual seja a da irredutibilidade das doenças cardiovasculares. Stallones ${ }^{12}$, Gordon e Thom ${ }^{4}$ e outros ${ }^{2}$ mostraram que, em vários países desenvolvidos, como os Estados Unidos, Inglaterra, Canadá e Austrália, desde o começo do século, as doenças cérebrovasculares vêm declinando rapidamente e que, desde a década de 50 a 60 , o mesmo vem acontecendo com as doenças coronarianas.

Devem ser lembrados os fatores de risco mais importantes e conhecidos para esses dois grupos de doenças. A hipertensão arterial é o fator mais importante: $80 \%$ das mortes por acidente vascular cerebral estão associadas à hipertensão arterial, enquanto que $40 \%$ das mortes por doenças coronarianas se acompanham de hipertensão. Além disso, a doença hipertensiva causa diretamente a morte de $5 \%$ dos que morrem por doenças cardiovasculares. Deste modo, podemos estimar que pelo menos $40 \%$ dos óbitos por doenças cardiovasculares apresentavam hipertensão arterial possivelmente 
como sua causa primária, e que pelo menos um entre oito óbitos por todas as causas é devido à hipertensão.

Os outros fatores de risco para as doenças coronarianas são o fumo, a vida sedentária, a obesidade e a ingestão de gorduras saturadas. A própria distribuição na população desses fatores de risco explica, em parte, as diferenças urbano-rurais encontradas por diversos autores 11,13 .

Portanto, das quatro crenças enumeradas inicialmente, a única que ainda parece resistir à evidência empírica cuidadosa é a de que a urbanização está associada positivamente com as doenças cardiovasculares.

Tendo este princípio como uma das preocupações mais importantes, realizamos, em 1978, um estudo sobre hipertensão arterial, um dos fatores de risco mais importantes para as mortes de adultos, na população do Estado do Rio Grande do Sul. Esse estudo, de tipo naturalístico, seccional, foi feito numa amostra de domicílios já anteriormente utilizada pelo Estudo Nacional de Despesas Familiäres (Instituto Brasileiro de Geografia e Estatística, para permitir também o estudo de fatores dietéticos e o hábito do fumo, porém cuja abordagem não será feita aqui ${ }^{3}$.

Como a urbanização é um fenômeno histórico complexo, não pretendemos captar toda a questão com um estudo seccional. Limitamo-nos a estudar a associação entre o estar numa condição e o status da pressão arterial.

Partimos da idéia de que a urbanização está fortemente ligada ao comércio e à indústria. Por isso, dividimos o espaço geopolítico rio-grandense em quatro estratos básicos.

A) O município de Porto Alegre. A área de mais elevado grau de urbanização, onde o setor terciário da economia é mais moderno: cerca de três quartos de sua população economicamente ativa trabalham nesse setor.

B) O Cinturão Metropolitano. Onde a urbanização é menos acentuada, e metade das pessoas economicamente ativas trabalha no setor secundário, a indústria.

C) O Urbano do Interior. Menos urbanizado do que a capital (a maior cidade tinha 200 mil habitantes), mas também com cerca de três quartos da população economicamente ativa no setor terciário, menos moderno todavia.

D) O Rural do Interior. Onde $90 \%$ da população economicamente ativa estão ligados ao setor primário, de agricultura e pecuária.

A descrição dos resultados nos permitirá avançar em aspectos relacionados à gênese dos diferenciais urbano-rurais nas doenças cardiovasculares.

A prevalência de hipertensão arterial foi significativamente mais elevada no Cinturão Metropolitano do que nos 
demais estratos e significativamente mais baixa no Interior Rural, também em relação aos demais. Porto Alegre e o Urbano do Interior apresentavam níveis semelhantes e intermediários. Esses resultados poderiam estar consolidando a afirmativa de que a industrialização está na raiz do problema, através do maior estímulo à migração, que seria fator determinante de hipertensão.

TABELA I

Prevalência ${ }^{1}$ de hipertensão arterial nos estratos geoeconômicos do Rio Grande do Sul, 1978

\begin{tabular}{|c|c|c|c|}
\hline Estratos & Prevalência (\% & & \\
\hline Cinturão Metropolitano & $13,76-$ & $b^{-} \mid a$ & \\
\hline Porto Alegre & $12,32-$ & NS & \\
\hline $\begin{array}{l}\text { Interior Urbano } \\
\text { Interior Rural }\end{array}$ & $\begin{array}{c}11,45- \\
9,25\end{array}$ & $a^{-1}$ & \\
\hline
\end{tabular}

$a=$ diferença significativa a $1 \%$

$\mathrm{b}=$ diferença significativa a $5 \%$

NS = diferença não-significativa

${ }^{1}$ Prevalência padronizada por idade e sexo pelo método indireto. (Padrão: taxas para o estado do Rio Grande do Sul).

Classificados os indivíduos de acordo com o fato de serem migrantes dentro do estrato ou entre estratos, bem como em relação à direção da migração, os resultados mostram que os naturais e os migrantes de cada estrato não apresentavam niveis tensionais diferentes ${ }^{7}$. Estes níveis parecem depender da direção da migração, isto é, os migrantes, independente da área de origem, adotam os níveis tensionais dos naturais residentes da área para onde se dirigem. Pela análise do tempo de migração, verificamos que esse processo não é imediato, tudo se passando como se fenômenos ambientais influenciassem os níveis tensionais.

As prevalências por setor econômico coincidem com as esperadas através dos estratos geoeconômicos. Surpreendentemente, porém, o setor terciário não se comportou de forma diferente do setor secundário em relação à pressão arterial. Já no setor primário, realmente, a prevalência de hipertensão é mais baixa do que nos demais. E, mais ainda, essa característica não explica os diferenciais de pressão entre os estratos.

A outra característica das pessoas que é de interesse estudar e que se relaciona com o processo produtivo é a posição na ocupação. Embora nosso estudo permitisse uma classificação mais complexa, vamos mostrar aqui os resultados segundo categorias dicotomicas, construídas de acordo com 
a relação de trabalho, a educação e o tamanho da propriedade rural 5 .

Nas atividades urbanos, o grupo A é constituído de proprietários dos meios de produção e de profissionais de nível superior de escolaridade, enquanto que o grupo B é formado pelos demais trabalhadores. Na área rural do Rio Grande do Sul, o corte foi feito em função do tamanho da propriedade: os que possuem ou exploram 50 hectares ou mais de terra constituem o grupo A, enquanto que o grupo B é formado pelos pequenos proprietários e trabalhadores rurais. Aqui mais do que nunca os resultados podem parecer surpreendentes. A tabela II mostra que os grupos B de qualquer um dos três setores econômicos apresentam médias de pressão sistólica quatro ou mais milímetros de mercúrio mais al tas do que os indivíduos dos grupos A. Isto significa, em termos correspondentes, que a prevalência de hipertensão arterial é de duas a três vezes maior nos trabalhadores dos setores econômicos terciário e secundário do que nos proprietários e outros segmentos privilegiados.

TABELA II

Médias ajustadas ${ }^{1}$ das pressões arteriais dos grupos sócio-econômicos - RGS, 1978

\begin{tabular}{lcccr}
\hline Setor Econômico & $\begin{array}{c}\text { Posição } \\
\text { no } \\
\text { Trabalho }\end{array}$ & Sistólica & Pressão & Diastólica \\
& & 129,5 & 72,4 & 545 \\
Nunca Trabalhou & & 124,2 & 67,5 & 99 \\
Primário & A & 128,4 & 70,6 & 735 \\
& B & 122,3 & 69,8 & 48 \\
Secundário & A & 129,9 & 73,0 & 804 \\
& B & 123,6 & 71,9 & 208 \\
Terciário & A & 128,3 & 72,7 & 1808 \\
& B & & \\
\hline
\end{tabular}

Análise de Variância

$\begin{array}{llll}\text { Sistólica: } & \mathrm{F}_{6 ; 4233}=4,65 & \mathrm{p}<0,001 & \mathrm{R}^{2} \text { total }=0,316 \\ \text { Diastólica: } & \mathrm{F}_{6 ; 4233}=5,93 & \mathrm{p}<0,001 & \mathrm{R}^{2} \text { total }=0,232\end{array}$

${ }^{1}$ Por idade, quetelet $(\log )$, circunferência do braço, pulso radial, razão sódio/creatinina $(\mathrm{log})$, sexo e cor da pele (regressão linear múltipla).

${ }^{2}$ A) Proprietários, arrendatários, parceiros e parentes com 50 hectares ou mais (Primário) ou donos e profissionais de nível superior (Secundário e Terciário).

B) Demais trabalhadores.

A diferença, embora menor, no meio rural teve a mesma direção. Porém, mais que tudo isso, controlando-se os resultados por esse fator sócio-econômico, através de modelos lineares aditivos que levam em consideração também outros fatores relevantes (Tabela III), as diferenças entre as áreas 
urbanas (Porto Alegre e Interior Urbano) e a área rural desaparecem. A prevalência no Cinturão Metropolitano também se aproxima bastante das demais, mas permanece ainda um pouco mais alta. A persistência de um nivel tensional mais elevado nos residentes no Cinturão Metropolitano poderia ser explicada através da metodologia utilizada para a análise, pois o método de ajustamente tem limitações por ser linear, sem levar em consideração possíveis in terações. Entretanto, outras explicações podem ser aludidas, como uma de ordem teórica: o excesso de risco ao nível coletivo ultrapassa a composição dos riscos individuais; e outra, ligada a fatores de risco específicos, como a população ambiental por resíduos industriais.

TABELA III

Médias ajustadas ${ }^{1}$ das pressões arteriais dos estratos geoconômicos - RGS, 1978

\begin{tabular}{|c|c|c|c|c|c|c|c|c|}
\hline \multirow[t]{2}{*}{ Pressão } & \multirow{2}{*}{\multicolumn{2}{|c|}{ Estrato }} & \multirow[t]{2}{*}{ Média } & & \multicolumn{2}{|c|}{ Contraste $^{2}$} & \multirow{2}{*}{ (C) } & \multirow{2}{*}{$\begin{array}{c}\mathrm{n} \\
\text { (D) }\end{array}$} \\
\hline & & & & & & & & \\
\hline \multirow[t]{4}{*}{ Sistólica } & (A) & Cinturão Metropolitano & 130,5 & \multirow[t]{4}{*}{$\mathrm{X}$} & * & $* * *$ & ** & 1092 \\
\hline & (B) & Porto Alegre & 128,4 & & $\mathrm{X}$ & NS & NS & 1036 \\
\hline & (C) & Interior Urbano & 127,4 & & & $\mathrm{X}$ & NS & 1088 \\
\hline & (D) & Interior Rural & 127,3 & & & & $X$ & 1031 \\
\hline \multirow[t]{4}{*}{ Diastólica } & (A) & Cinturão Metropolitano & 73,7 & \multirow[t]{4}{*}{$\mathrm{X}$} & * & *** & $* * *$ & 1092 \\
\hline & (B) & Porto Alegre & 72,3 & & $\mathrm{X}$ & NS & NS & 1036 \\
\hline & (C) & Interior Urbano & 71,5 & & & $\mathrm{X}$ & NS & 1088 \\
\hline & (D) & Interior Rural & 71,0 & & & & $\mathrm{X}$ & 1031 \\
\hline
\end{tabular}

Análise de Variância

Sistólica: $F 3 ; 4230=5,25 \quad \mathrm{p}<0,01 \quad \mathrm{R}^{2}$ total $=0,318$

Diastólica: $F \quad 3 ; 4230=7,40 \quad \mathrm{p}<0,001 \mathrm{R}^{2}$ total $=0,236$

${ }^{1}$ Por idade, quetelet (log), circunferência do braço, pulso radial, razão sódio/creatinina $(\log )$, sexo, cor da pele e setor econômico posição no trabalho (regressão linear múltıpla)

$\begin{array}{rl}{ }^{2} \text { NS } & \mathrm{P}>0,05 \\ * & \mathrm{p}<0,05 \\ * * & \mathrm{p}<0,01 \\ * * * & \mathrm{p}<0,001\end{array}$

Para apoiar esses achados no Rio Grande do Sul, podemos aduzir ainda as observações feitas também pelo nosso grupo de estudos, em Volta Redonda, no Estado do Rio de Janeiro, no final do ano de $1979^{1}$. Aí, com metodologia e instrumental semelhantes aos utilizados na pesquisa anterior, pudemos comparar apenas grupos ocupacionais urbanos. Porém, dessa vez não nos causou nenhuma surpresa o fato de que o grupo socialmente mais privilegiado, de proprietários e profissionais de nível universitário, mostrou os índices mais baixos de pressão e prevalência de hipertensão. 
Em Volta Redonda, os piores indicadores foram apresentados pelas pessoas com atividades subalternas da prestação de serviços, o que pode ser explicado por particularidades locais de determinação histórica da composição da população, de acordo com sua inserção no processo produtivo ${ }^{6}$.

Esses resultados indicam que seria a maior ou menor proporção de pessoas assalariadas nos setores secundário e terciário que determinaria o nível de hipertensão em uma população. Em outras palavras, ou é a concentração da riqueza e, portanto, do acesso aos bens materiais que determina fortemente a pressão arterial, ou é a submissão e a repressão na vida social dos indivíduos, ou ambos. Entretanto, a mediação entre esses determinantes mais amplos e o estado tensional (arterial) não está elucidada e, mais provavelmente, não está ligada a essa categoria simplificada e imprecisa chamada estresse.

Em conclusão, quisemos com o presente relato chamar a atenção para o fato de que o meio urbano é um meio descontínuo socialmente, e que essa descontinuidade, se incorporada aos estudos biológicos, dá maior poder explicativo às variações constitucionais dos indivíduos. O nosso meio urbano concentra poder, autoridade e riqueza nas mãos de poucos e concentra miséria e doença nas vidas de muitos. Além disso, as áreas industriais concentram poluentes tanto no nível de meio interno, como as fábricas e as casas, como no meio externo, ainda de difícil avaliação em relação à saúde.

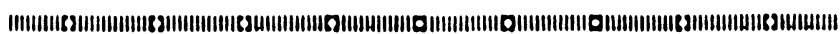

It is discussed four very frequent beliefs regarding cardiovascular diseases, especially that they are harmful consequences from urbanization and they are specifically linked to rich people. However, our data showed that the size of the proportions of persons employed in secondary or tertiary sectors of the economy will determine hypertension levels in a given population. These findings were the result of analyses of studies carried out by the authors in Rio Grande do Sul and Volta Redonda.

Moreover, concentration of wealth, easy access to goods and/or submission and repression in one's social life have a strong influence in everyone's blood pressure.

\section{REFERÊNCIAS BIBLIOGRÁFICAS}

1. ARAÛJO, J.W.G. Hipertensão arterial em grupos sócio-ocupacionais de Volta Redonda-RJ. Rio de Janeiro, 1984. [Tese de Mestrado - ENSP] .

Cadernos de Saúde Pública, R.J., 1 (3): 305-312, jul/set, 1985. 
2. BARKER, D.J.P. \& ROSE, G. Epidemiology in medical practice. Edinburgh, Churchill Livingstone, 1976.

3. COSTA, E.A. A cross-sectional survey of blood pressure in Rio Grande do Sul - Brazil. London, 1981. [PhD Thesis - Faculty of Medicine, University of London].

4. GORDON, R. \& THOM, T. The recent decrease in CHD mortality. Prev. Med., 4: 115, 1978.

5. KLEIN, C.H. Hipertensão arterial nos estratos geo-econômicos do Rio Grande do Sul. Rio de Janeiro, 1984. [Tese de Mestrado - ENSP] .

6. KLEIN, C.H. \& ARAÜJO, J.W.G. Fumo, bebida alcoólica, migração, instrução, ocupação, agregação familiar e pressão arterial em Volta Redonda. Cad. Saúde Públ., 1 (2): 160, 1985.

7. LEAL, M.C. Migrações internas e pressão arterial no Rio Grande do Sul. Rio de Janeiro, 1981. [Tese de Mestrado - ENSP] .

8. MARMOT, M.G.; ROSE, G.; SHIPLEY, M. \& HAMILTON, P.J.S. Employment grade and coronary heart disease in british civil servants. J. Epidemiol. \& Comm. Health, 32: $244,1978$.

9. PUFFER, R.R. \& GRIFFITH, G.W. Patterns of urban mortality. Washington, PAHO, 1968. [Scientific publication, 151].

10. ROSE, G. \& MARMOT, M. Social class and coronary heart disease. Br. Heart J., 45: 13, 1981.

11.SHAPER, A.G. Cardiovascular disease in the tropics - II: Blood pressure and hypertension. Br. Heart J., 3: 805, 1972.

12. STALLONES, R.A. The rise and fall of ischaemic heart disease. Sci. Am., 243: 43, 1980.

13. STAMLER, J.; KJELSBERG, M.; HALL, Y. \& SCOTH, N. Epidemiologic studies on cardiovascular-renal disease - I and II: Analysis of mortality by age-race-sex-occupation and by place of residence including urban-rural comparison. J. Chron. Dis., 12: 440, 1960. 